\title{
Development of an MDO platform for aero-structural interactions
}

\author{
Zhe $\mathrm{Hu}^{\mathrm{a}}$, Yuexi Xiong, Jingwu He and Yang Pan \\ School of Aeronautical Science and Engineering, Beijing University of Aeronautics and Astronautics, Beijing, China
}

\begin{abstract}
An interactive MDO platform for multivariate and high coupling aero-structural interactions is presented in this paper. Programs based on RSM and GA are utilized as optimization solver, which is connected with CAD/CAE software by a friendly GUI. To generate and modify CAD models in the analysis and optimization process, CATIA Automation Technique is adopted to create a CAD model generator. During the analysis process, high-fidelity models in both disciplines are used. For aircraft wing MDO problems, optimal wing shape and structural configuration can be obtained using this platform in the preliminary design phase. While the current platform is aiming at solving Aero-Structural problems in the preliminary design phase of UAV components, it also provides a reference solution for other related MDO problems. Two cases are provided to verify the validity and practicability of this platform. Besides the design ideas and techniques, this paper also demonstrates its further development capabilities.
\end{abstract}

\section{Nomenclature}

$\begin{array}{ll}\mathrm{C}_{\mathrm{l}} & =\text { Lift coefficient } \\ \mathrm{C}_{\mathrm{l}}^{\text {Baseline }} & =\text { Lift coefficient of the initial design } \\ \mathrm{C}_{\mathrm{d}} & =\text { Drag coefficient } \\ \mathrm{C}_{\mathrm{d}}^{\text {Baseline }} & =\text { Drag coefficient of the initial design } \\ \mathrm{L} / \mathrm{D} & =\text { Lift to drag ratio } \\ \Lambda & =\text { Sweep angle } \\ \lambda & =\text { Taper ratio } \\ \mathrm{U}_{\max } & =\text { Maximum displacement of the wing } \\ \mathrm{M} & =\text { Structural Mass } \\ \mathrm{rsb} & =\text { Thickness of the rear spar flanges } \\ \mathrm{fsb} & =\text { Thickness of the front spar flanges } \\ \mathrm{pt} & =\text { Thickness of the spar plates } \\ \mathrm{st} & =\text { Thickness of the skin }\end{array}$

${ }^{a}$ Corresponding author: huzhebuaa@163.com 


\section{Introduction}

With the continuous development of the Computer Aided Engineering (CAE) Technology, high precision analysis methods based on Computational Fluid Dynamics (CFD) and Computational Structure Mechanics (CSM) can be applied during the preliminary design phase of the Unmanned Aerial Vehicles (UAV) to obtain more precise simulation results [1]. Wing, as important part of an $\mathrm{UAV}$, is closely related to the aero-structural interaction issues. And the design of a wing is a comprehensive issue connected with various disciplines. Recent years, researchers have been working on the development of Multidisciplinary Design Optimization (MDO) frameworks and platforms in which high precision modeling, analysis techniques are tightly integrated [2-5]. Therefore, it is evident that a platform with friendly GUI which can help to deal with MDO problems that considering aerostructural interactions is essential for aircraft designers and engineers. The MDO platform is consist of the modeling, analysis, optimization modules and the data exchanger. Equipped with a graphic user interface, the platform is able to provide various options for modeling, analysis and optimization, and can seek for an optimal configuration for preliminary wing design issues.

\section{CAD modeling module}

Parametric modeling is essential to be applied in the pre-processing stage. Especially in the optimization stages, design variables related to parametric modeling should be modified to generate new design points. Therefore, on one hand, parametric modeling with a graphic interface can help to generate, modify, and check the model in a more efficient way. On the other hand, its property makes it possible to be easily applied in the optimization algorithms. Computer Aided Three-dimensional Interactive Application (CATIA), known as one of the most common used Modeling software in aviation industry, provides a programming technique named CATIA Automation to create and modify models in form of VBSripts. The generation process of the model is demonstrated in Figure 1.

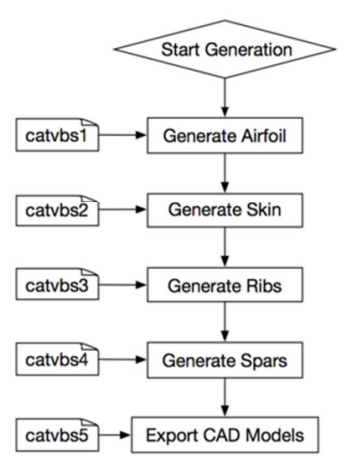

Figure 1. CAD Model Generation Process.

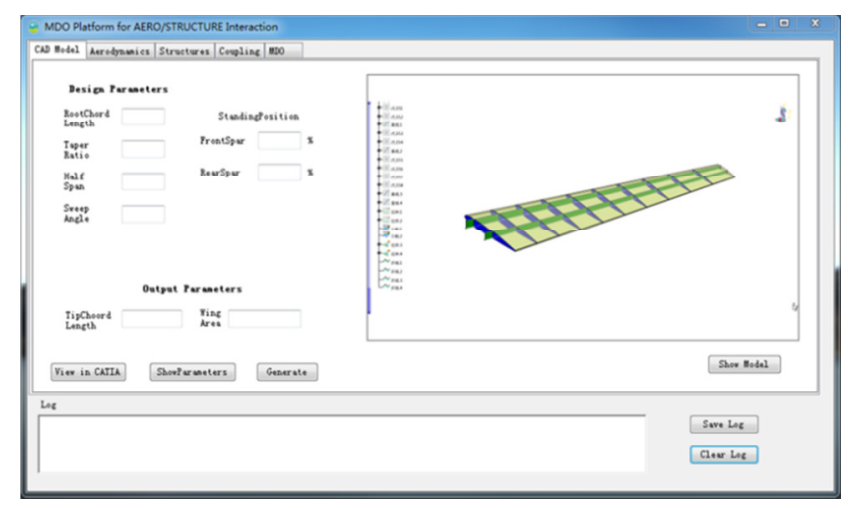

Figure 2. CAD Modeling Module.

Specifically, the generation form of the CAD Model is in a sequential way in which the skin, ribs, spars are generated in order. Different kinds of wing structures can be generated by modular scripts to expand the model database. The CAD parametric modeling process is aimed to generate models for both aerodynamics and structural analysis processes. The interface of CAD modeling module is shown in Figure 2. Wing shape parameters can be quickly modified to generate CAD models through the GUI. In addition, a log Window is provided to record and show the important information and user operations, and it can help to check the input and intermediate process information when error occurs. 


\section{Analysis module}

The analysis workflow is shown in Figure 3. The analysis process begins with the aerodynamic mesh generation. After the aerodynamic analysis, pressure data generated by interpolation method can be obtained after the aerodynamic analysis process. Python script is applied to extract the load data from '.dat' file and create an '. inp' file for ABAQUS in which the finite elements information, material properties, boundary conditions and aerodynamic loads are recorded. Once the analysis process is completed, the analysis results for each discipline can be obtained from the interface.

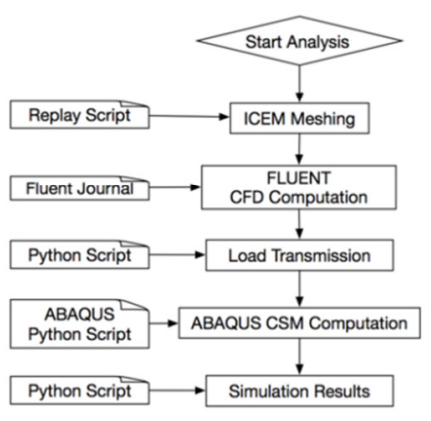

Figure 3. Analysis Workflow.

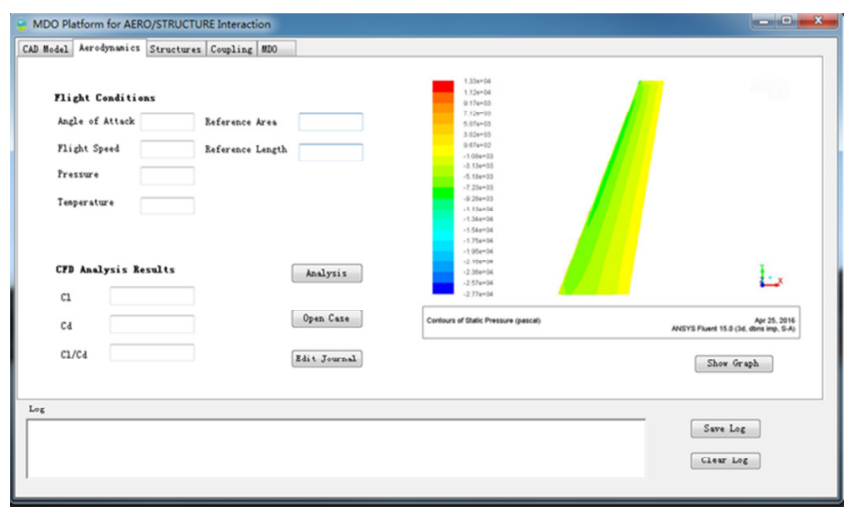

Figure 4. Aerodynamic Analysis Module.

\subsection{Aerodynamic analysis}

Aerodynamic analysis method based on CFD is able to compute the aerodynamic performance through a high precession way. In the interface of aerodynamic analysis module shown in Figure 4, flight conditions can be modified through the GUI to obtain the aerodynamic performance of the wing.

During the analysis process, Spalart-Allmaras viscosity model is adopted to simulate the turbulence of the flow field. Flux Type of Roe-FDS and Implicit formulation are chosen as the solution method [6]. A wing mesh for CFD computation is shown in Figure 5. It is generated through an ICEM replay script and has the $\mathrm{O}$ type grid topology. The automatic aerodynamic analysis process is driven by a '. journal' script of FLUENT. When the analysis process is completed, a python script is used to extract the flight performance parameters and generate an '. inp' file for the following structural analysis.

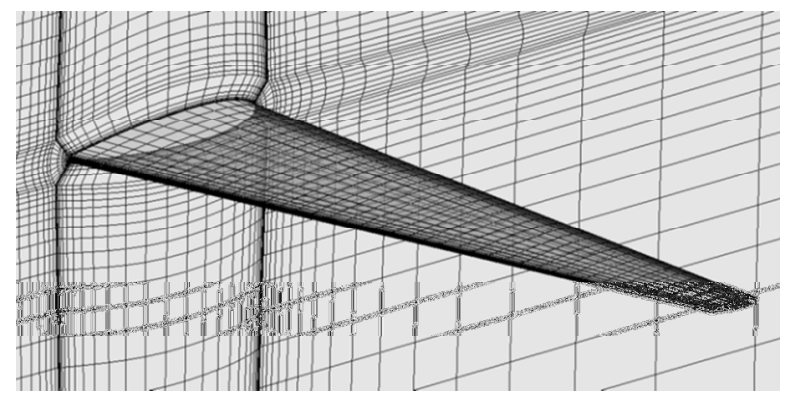

Figure 5. Aerodynamic Analysis Module.

\subsection{Structural analysis}

Patran is often chosen as the structural analyzer for wing MDO problems [7-9]. In this paper, in order to create CSM model more efficiently, ABAQUS is selected as the structural pre-processer and 
analyzer. The structural analysis simulation is based on the finite element method (FEM). The interface of structural analysis module is shown in Figure 6. Structural parameters and material properties can be modified through the interface to get the maximum stress and displacement on each component. In addition, stress and displacement nephograms, structural mass and other simulation results can be obtained. Moreover, the platform can also provide more detailed analysis information such as the analysis database and the original project files.

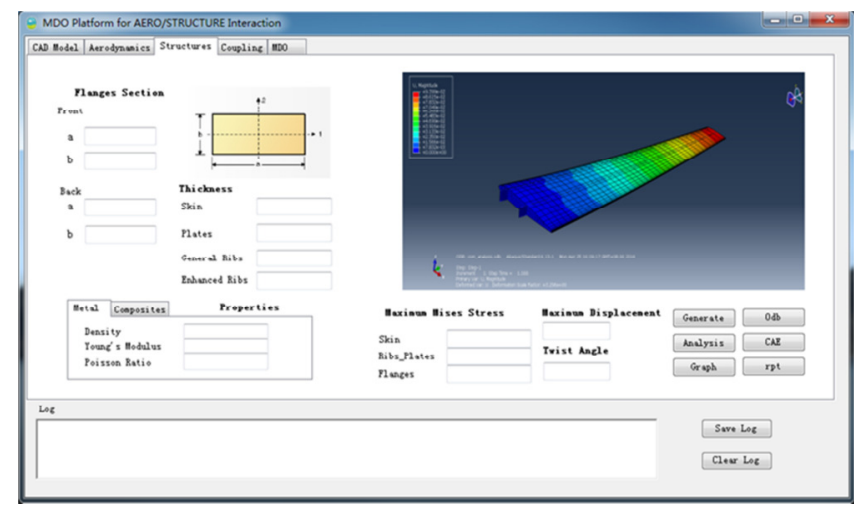

Figure 6. Structural Analysis Module.

\section{Aero-structural coupling}

Aero-structural interactions have a notable influence on the aerodynamic performance of the wing. The deformation and its impacts on external flow should be taken into consideration. The max displacement on a wing tip is chosen as a characteristic variable. The changing tendency of the maximum displacement on a wing tip is shown in Figure 7.

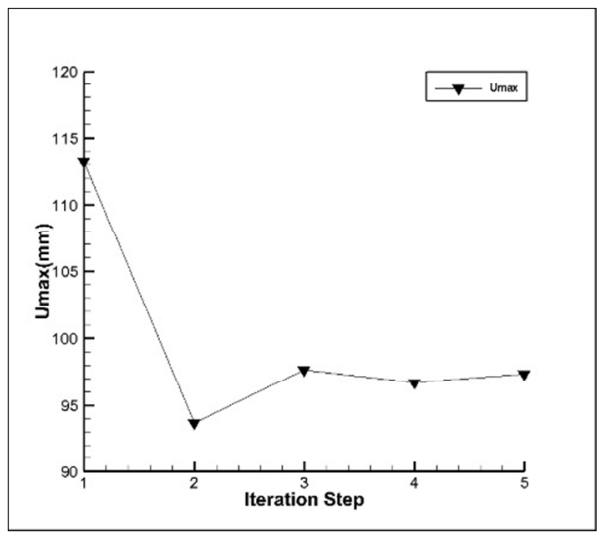

Figure 7. Variation of $U_{\max }$ with Iteration Step.

The solution is converged after 5 iterations according to the convergence criterion demonstrated as below:

$$
\left|\frac{U_{\max }^{i}-U_{\max }^{i-1}}{U_{\max }^{i}}\right|<0.01
$$

Where $U_{\text {max }}^{i}$ is the maximum displacement of the wing tip in the $i^{\text {th }}$ iteration step. A series of case have been tested to come to a conclusion that the computation results will be converged after 3-5 times of iterations. The impact of the coupling effect on lift coefficient $(\mathrm{Cl})$ is shown in Figure 8. 


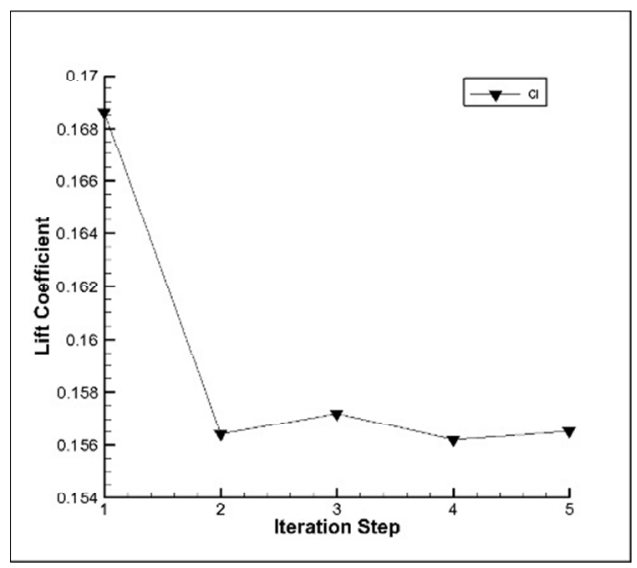

Figure 8. Variation of $\mathrm{Cl}$ with Iteration Step.

Compared with the case of a rigid wing, the lift coefficient decreases about $7 \%$. Therefore, for the optimization of aerodynamic performance, it is of great significance to consider the effects of aerostructural interactions in order to establish a more actual and precise simulation model.

\section{Multidisciplinary design optimization}

\subsection{MDO Architecture}

MDO is a research field which has been growing rapidly as the analysis and approximation techniques are developed. Wing design is a representative issue since the interactions of disciplines like aerodynamics and structural theory have to be taken into account. Sharing many similarities with Collaborative Optimization (CO) [10], the MDO architecture integrated in the platform is demonstrated in Figure 9.

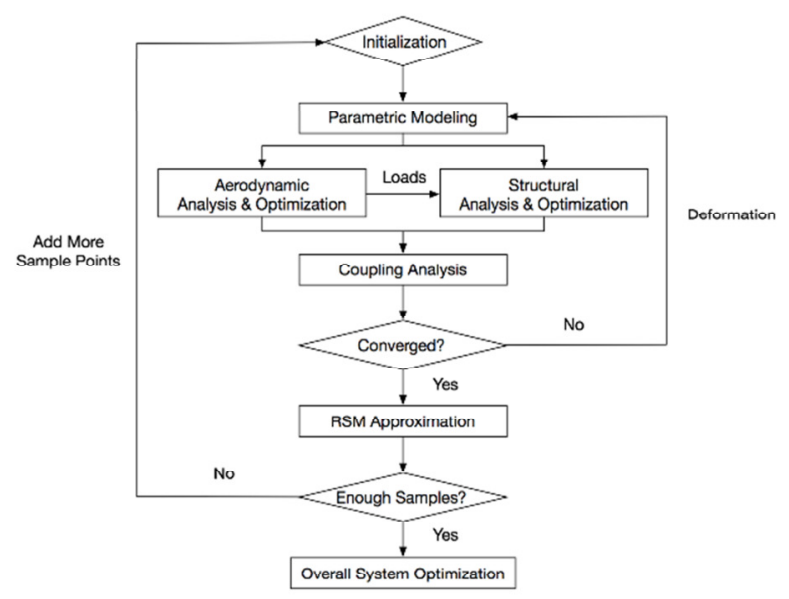

Figure 9. MDO Process.

The optimization process has two levels. One is the overall system optimization in which the global design variables that can influence the optimization process in all subsystems are defined, the other is the subsystem optimization in which local design variables are determined for each discipline after the initialization of the global design. After the optimization processes in all subsystems are finished, the coupling analysis process is executed to obtain the objective variables. Since the 
optimization cost without approximation techniques is huge, Respond Surface Method (RSM) [3] is integrated in the platform to establish the system level surrogate model and reduce the optimization time.

\section{Case study}

\subsection{Case 1: Sequential optimization}

The wing in case 1 flies at the speed of Ma 0.4, and its AOA is $2^{\circ}$. Semi-coupling method is applied in the optimization process of case 1 . Specifically, the aerodynamic load is defined as the decoupling variable between the two disciplines, and the impacts on the external flow field due to structural deformation have not been taken into consideration during the optimization process. The aerodynamic optimization is performed in the first phase. Then the optimal wing shape is confirmed and the structural optimization and coupling analysis are executed in the second phase. The design target of case 1 is to maximize the lift to drag ratio during the aerodynamic optimization process and minimize the structural weight of the wing in the structural optimization phase. Six structural design variables are selected through the Design of Experiment (DOE). The mathematic model of the optimization problem is described as follows:

$$
\left.\begin{array}{lc}
\text { Maximize: } & \begin{array}{l}
L / D \\
\text { Subject to: }
\end{array} \\
C_{l} \geq C_{l}^{\text {Baseline }}, X_{\text {aero }}^{\min } \leq X_{\text {aero }} \leq X_{\text {aero }}^{\max }
\end{array}\right\} \text { Aerodynamic Optimization }
$$

Where $X_{\text {aero }}$ is the aerodynamic design vector which is constrained by the design limits. It consists of the sweep angle and taper ratio of the wing. And the $X_{\text {struc }}$ is the structural design vector which includes the skin thickness, spar section parameters and the thickness of the spar plates. $\sigma_{\text {allowable }}$ represents the allowable stress of the structure and $u_{\text {allowable }}$ stands for the allowable structural displacement. RSM are both applied for each discipline to create approximation models after the precision test. Fifty-eight design points generated by Optimal Latin Hypercube [11] are used to create RS surrogate model for aerodynamic analysis while 50 design samples generated by Optimal Latin Hypercube Design are applied to create RS surrogate model for structural analysis. The results of the prediction accuracy evaluation for respond surface models are shown in Table 1.

Table 1. Prediction Accuracy Evaluation for RSM in Case 1.

\begin{tabular}{|c|c|c|}
\hline Items & $\mathbf{R}^{\mathbf{2}}$ & $\mathbf{R M S E}$ \\
\hline $\mathrm{L} / \mathrm{D}$ & 0.985 & 0.025 \\
\hline $\mathrm{M}$ & 0.999 & 0.009 \\
\hline
\end{tabular}

During the optimization process, Sequential Quadratic Programming (NLPQL) [12] is selected as the optimization algorithm and integrated in the platform. The final results are shown in Table 2.

Table 2. Optimization Results of Case 1.

\begin{tabular}{|c|c|c|c|c|c|c|}
\hline Items & $\begin{array}{c}\text { Variables } \\
\text { Constraints }\end{array}$ & $\begin{array}{c}\text { Initial } \\
\text { Design }\end{array}$ & $\begin{array}{c}\text { Optimal } \\
\text { Design(RSM) }\end{array}$ & $\begin{array}{c}\text { Optimal } \\
\text { Design(Actual) }\end{array}$ & $\begin{array}{c}\text { Variation(Initial } \\
\text { vs Optimal) }\end{array}$ & $\begin{array}{c}\text { Error(RSM } \\
\text { vs Actual) }\end{array}$ \\
\hline$\Lambda$ & {$[15,25]$} & 25 & 20.29 & N/A & $-18.84 \%$ & N/A \\
\hline$\lambda$ & {$[0.25,0.35]$} & 0.3 & 0.25 & N/A & $-28.6 \%$ & N/A \\
\hline $\mathrm{rsb}$ & {$[2,3.5]$} & $3 \mathrm{~mm}$ & $2.02 \mathrm{~mm}$ & N/A & $-32.6 \%$ & N/A \\
\hline
\end{tabular}




\begin{tabular}{|c|c|c|c|c|c|c|}
\hline $\mathrm{fsb}$ & {$[2,3.5]$} & $3 \mathrm{~mm}$ & $3.49 \mathrm{~mm}$ & N/A & $+16.3 \%$ & N/A \\
\hline $\mathrm{st}$ & {$[1,2]$} & $1 \mathrm{~mm}$ & $1.1 \mathrm{~mm}$ & N/A & $+10 \%$ & N/A \\
\hline $\mathrm{pt}$ & {$[2,4]$} & $3 \mathrm{~mm}$ & $3.51 \mathrm{~mm}$ & N/A & $+17 \%$ & N/A \\
\hline$C_{l}$ & {$[0.1705, \infty]$} & 0.1705 & 0.1887 & $0.1832^{*}$ & $+3.9 \%$ & N/A \\
\hline$C_{d}$ & N/A & 0.0222 & 0.0211 & $0.0225^{*}$ & $-2.1 \%$ & N/A \\
\hline $\mathrm{L} / \mathrm{D}$ & N/A & 7.65 & 8.54 & $8.14 *$ & $+6.4 \%$ & N/A \\
\hline $\mathrm{M}$ & N/A & $18.62 \mathrm{~kg}$ & $20.11 \mathrm{~kg}$ & $19.93 \mathrm{~kg}$ & $+7.03 \%$ & $0.9 \%$ \\
\hline
\end{tabular}

*Coupling analysis has been performed.

Compared with the baseline design, the lift to drag ratio of the optimal design increases about $0.83 \%$. However, the structural weight increases about $7.03 \%$. The optimization results illustrate that the sequential optimization process from the aerodynamics discipline to the structures discipline is failed to find an optimal design point satisfying the design targets of all the disciplines.

\subsection{Case 2: Multidisciplinary design optimization}

Tight-coupling method is applied in case 2. Specifically, during the whole optimization process, aerodynamic load and the structural deformation are both taken into account and defined as the decoupling variables. The initial design conditions are the same as case 1 . That means the influence of the aerodynamic loads and the impacts on external fluid flow caused by the structural deformation have been considered in the optimization process. The optimization workflow is shown in Figure 9. In this case, the optimization process is divided into 2 levels. One is the overall system level in which global design vectors are determined. The other is the subsystem level which includes the aerodynamic analysis (Note: No variables are defined to optimize in aerodynamic subsystem) and the structural optimization. The mathematic model of the optimization problem is described as follows:

\section{System Level Optimization}

$$
\left.\begin{array}{lc}
\text { Maximize: } & F\left(X_{\text {sys }}\right)=W_{1} * C_{\text {mass }}+W_{2} * C_{l / d} \\
\text { Subject to : } & C=\left|Y_{\text {sys } 1}-Y_{\text {aero }}\right|+\left|Y_{\text {sys } 2}-Y_{\text {struc }}\right|=0
\end{array}\right\}
$$

Where $X_{\text {sys }}$ represents the design vector of the overall system level. The consistency constraint $\mathrm{C}$ ensures the supplementary vector $Y_{\text {sys } 1}\left(\right.$ or $Y_{\text {sys } 2}$ ) is equal to the coupling vector $Y_{\text {aero }}$ (or $Y_{\text {struc }}$ ). Weighting method is applied in the system level optimization to convert the multi-objective optimization problem to a single objective optimization problem. Also in the equation above, $W_{1}$ is the weight coefficient of the structural mass and $W_{2}$ is the weight coefficient of the lift to drag ratio. In this case, they are both set as 0.5 . $C_{\text {mass }}$ represents the decrease coefficient of structural weight while $C_{l / d}$ stands for the increase coefficient of lift to drag ratio, both of which are determined as follows:

$$
\left\{\begin{array}{c}
C_{\text {mass }}=\frac{M_{\text {Baseline }}-M_{o p t}}{M_{\text {Baseline }}} \\
C_{l / d}=\frac{L / D_{o p t}-L / D_{\text {Baseline }}}{L / D_{\text {Baseline }}}
\end{array}\right.
$$

The mathematic model of subsystem optimization (Structural Discipline) can be described as follows: 


$$
\begin{array}{cc}
\text { Minimize: } & M \\
\text { Subject to: } & \sigma_{\max } \leq \sigma_{\text {allowable }}
\end{array} \quad, X_{\text {struc }}^{\min } \leq X_{\text {struc }} \leq X_{\text {struc }}^{\max }
$$

When the optimization process in all subsystems are completed, the coupling analysis will be performed. Then statistical data of single design point is obtained. In the overall system level, 48 design points are used to generate the RS model to perform the overall system optimization. The prediction accuracy evaluation for RSM is shown in Table 3 and precision of the RS Model is high enough to replace the original CAE analysis process.

Table 3. Prediction Accuracy Evaluation for RSM in Case 2.

\begin{tabular}{|c|c|c|}
\hline Items & $\mathbf{R}^{\mathbf{2}}$ & $\mathbf{R M S E}$ \\
\hline $\mathrm{L} / \mathrm{D}$ & 0.998 & 0.012 \\
\hline $\mathrm{M}$ & 0.999 & 0.002 \\
\hline
\end{tabular}

Finally, the optimization results based on the 2-level MDO architecture is illustrated in Table 4. The L/D of the optimal wing has increased about $7.5 \%$ and the structure weight has decreased about $5.9 \%$. The optimization results demonstrate that the optimal wing shows better performance in both disciplines, while the prediction error of the surrogate model is less than $2 \%$ which proves the validity and practicability of the MDO platform. Compared to the results of case 1, the MDO architecture is more capable to seek the global optimal design.

Table 4. Optimization Results of Case 2.

\begin{tabular}{|c|c|c|c|c|c|c|}
\hline Items & $\begin{array}{c}\text { Variables } \\
\text { Constraints }\end{array}$ & $\begin{array}{c}\text { Initial } \\
\text { Design }\end{array}$ & $\begin{array}{c}\text { Optimal } \\
\text { Design(RSM) }\end{array}$ & $\begin{array}{c}\text { Optimal } \\
\text { Design(Actual) }\end{array}$ & $\begin{array}{c}\text { Variation(Initial } \\
\text { vs Optimal) }\end{array}$ & $\begin{array}{c}\text { Error(RSM } \\
\text { vs Actual) }\end{array}$ \\
\hline$\Lambda$ & {$[15,25]$} & 25 & 15.95 & N/A & $-36.2 \%$ & N/A \\
\hline$\lambda$ & {$[0.25,0.35]$} & 0.3 & 0.252 & N/A & $-28.0 \%$ & N/A \\
\hline $\mathrm{rsb}$ & {$[2,3.5]$} & $3 \mathrm{~mm}$ & $2 \mathrm{~mm}$ & N/A & $-33.3 \%$ & N/A \\
\hline $\mathrm{fsb}$ & {$[2,3.5]$} & $3 \mathrm{~mm}$ & $3.15 \mathrm{~mm}$ & N/A & $+5 \%$ & N/A \\
\hline $\mathrm{st}$ & {$[1,2]$} & $1 \mathrm{~mm}$ & $1 \mathrm{~mm}$ & N/A & $0 \%$ & N/A \\
\hline $\mathrm{pt}$ & {$[2,4]$} & $3 \mathrm{~mm}$ & $2.63 \mathrm{~mm}$ & N/A & $+12.6 \%$ & N/A \\
\hline$C_{l}$ & {$[0.1705, \infty]$} & 0.1705 & 0.1889 & 0.1865 & $+9.4 \%$ & $1.2 \%$ \\
\hline$C_{d}$ & N/A & 0.0222 & 0.0229 & 0.0227 & $-2.25 \%$ & $0.87 \%$ \\
\hline $\mathrm{L} / \mathrm{D}$ & N/A & 7.65 & 8.251 & 8.225 & $7.5 \%$ & $0.32 \%$ \\
\hline $\mathrm{M}$ & N/A & $18.62 \mathrm{~kg}$ & $17.63 \mathrm{~kg}$ & $17.52 \mathrm{~kg}$ & $-5.9 \%$ & $0.62 \%$ \\
\hline
\end{tabular}

\section{Further development possibilities}

The platform specifically designed for Wing MDO problems is able to seek an optimal wing configuration and provides modules for computation tests. The MDO platform has integrated the MDO architecture, high-precision simulation methods, approximation techniques and optimization algorithms by scripting language and packages all the modules through the GUI. The concept of modular design makes it a potential platform that can allow third-party users and programmers to quickly customize and enhance its functions. The architectures of different modules in this platform is illustrated in Figure 10. Owing to the concept of modular design and the standardization of this platform, the benefits are showed as follows. 


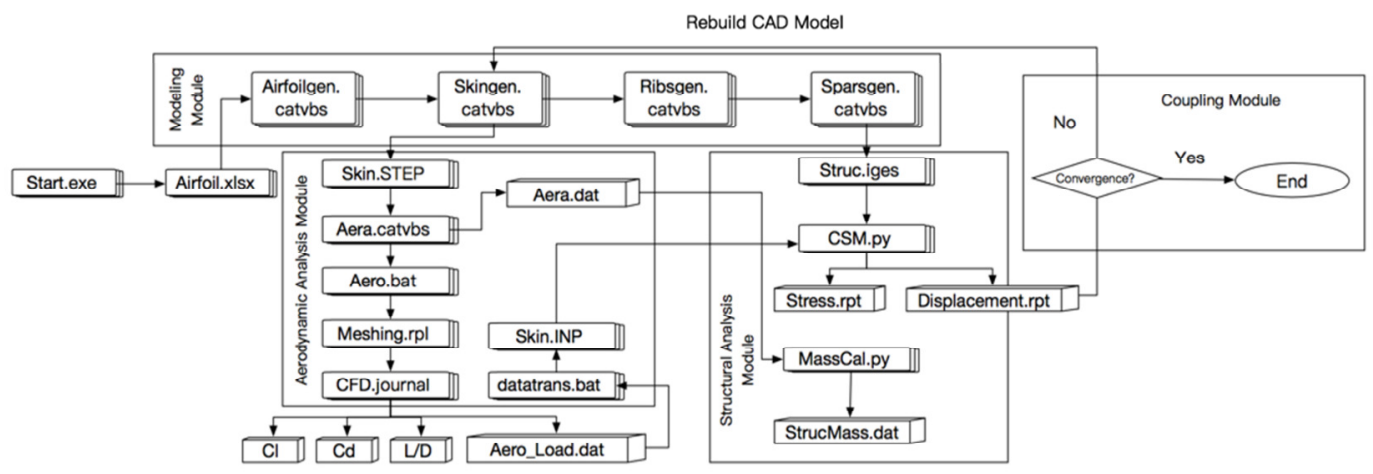

Figure 10. Architectures of different modules of the platform.

Firstly, various VBScripts are integrated in the CAD generator to create different inner structures. The wing structure database can be extended by integrating more CAD generation scripts in a efficient way. Secondly, because of the portability and interchangeability of the scripting language, the secondary development environment can be simple and neat. Third-party optimization algorithms and approximation techniques can be integrated by adding a simple corresponding script. Lastly, owing to the universal storage form of simulation data, it is easier for different post-processing tools to exact data from the database.

Original designed for wing MDO issues, the platform is being developed for more possibilities, allowing more disciplines, MDO architectures, optimization algorithms to be integrated in the future development. In terms of user interaction and data management, the platform will be improved to simplify the setup process and make the data storage normalized.

\section{References}

1. T.W. Simpson, V. Toropov, V. Balabanov, et al., 12th AIAA/ISSMO multidisciplinary analysis and optimization conference, 5, 10-12 (2008)

2. J. Martins, C. Marriage, Aiaalasme/ascelahs/asc Structures, Structural Dynamics, and Materials Conference (2007)

3. R.H. Myers and D.C. Montgomery, Technometrics, 38 (3), 284-286 (1996)

4. R. Paiva, Aiaa/issmo Multidisciplinary Analysis and Optimization Conference (2008)

5. L. Attanasi, A.D. Marco, F. Nicolosi, et al., Aiaa Applied Aerodynamics Conference (2015)

6. P. Spalart and S. Allmaras, La Recherche Aérospatiale, 439 (1), 5 -21 (1992)

7. T. Long, L. Liu, S. Zhou, et al., Aiaa/issmo Multidisciplinary Analysis and Optimization Conference (2008)

8. H. Jie and Y. Xiongqing, Comput Aid Eng., 22 (1), 16-20 (2013)

9. H. Jie, R. Wang, et al., TNUAA, 44 (4), 458-463 (2012)

10. R.D. Braun, A.A. Moore, and I.M. Kroo, 6th AIAA/USAF/NASA/ISSMO Symposium on Multidisciplinary Analysis and Optimization (2010)

11. J.S. Park, J Stat Plan Infer, 39 (1), 95-111 (1994)

12. K. Schittkowski, Ann Oper Res., 5 (1), 485-500 (1986) 